\title{
NUMERICAL SIMULATION OF THE SURFACE FLOW OF A COMPANION STAR IN A SEMI-DETACHED BINARY SYSTEM AND ITS APPLICATION TO GALACTIC SUPERSOFT X-RAY SOURCES
}

\author{
K. Oka, ${ }^{1}$ T. Matsuda,${ }^{1}$ I. Hachisu,${ }^{2}$ and H. M. J. Boffin ${ }^{3}$
}

We numerically simulate the surface flow of a gas-supplying companion star in a semidetached binary system and construct the Doppler map. Our numerical results indicate an eddy configuration composed of a $\mathbf{H}$ eudy, a L1-eddy, and a L2-eddy associated with high/low pressures on the surface of the companion star. We find that the formation of these eddies can be explained in terms of the astrostrophic wind. We apply our calculations to the Galactic supersoft X-ray source RX J0019.8+2156. This object is very attractive for the present study since the observed Doppler map might reflect the surface flow on the companion star. We have constructed the Doppler map of RX J0019.8+2156 and compare it with observations.

Although the knowledge on the surface flow of the companion star is important to understand the evolution of a binary system, there have been no detailed studies on this topic except for the work of Lubow \& Shu (1975). Their work is, however, semi-analytic, limiting the possibility to investigate the surface flow in detail. Numerical simulations are thus called for. Figures 1 show our numerical results for the case with an unit mass ratio. The remarkable feature is that three eddies, namely the H-eddy (around the pole), the L1-eddy (around the L1 point), and the L2-eddy (around the opposite side of the L1 point) appear on the companion's surface. The generation mechanism of these eddies is explained in terms of the astrostrophic wind and the dynamics of an eddy system (Oka et al. 2002). We think that the Heddy corresponds to the astrostrophic wind found by Lubow \& Shu.

We apply our calculations to RX J0019.8+2156, which is a very mysterious object. as: 1) although a low radial velocity component of the emission lines of He II $\lambda 4686$ is observed, the region from where this

\footnotetext{
${ }^{1}$ Department of Earth and Planetary Sciences, Kobe University, Kobe 657-8501, Japan (kazutaka@kobe-u.ac.jp).

${ }^{2}$ Department of Earth Science and Astronomy, University of Tokyo, Komaba, Meguro-ku, Tokyo 153-8902, Japan.

${ }^{3}$ European Southern Observatory, Karl-Schwarzschild-Str. 2, D-85748 Garching-bei-München, Germany.
}
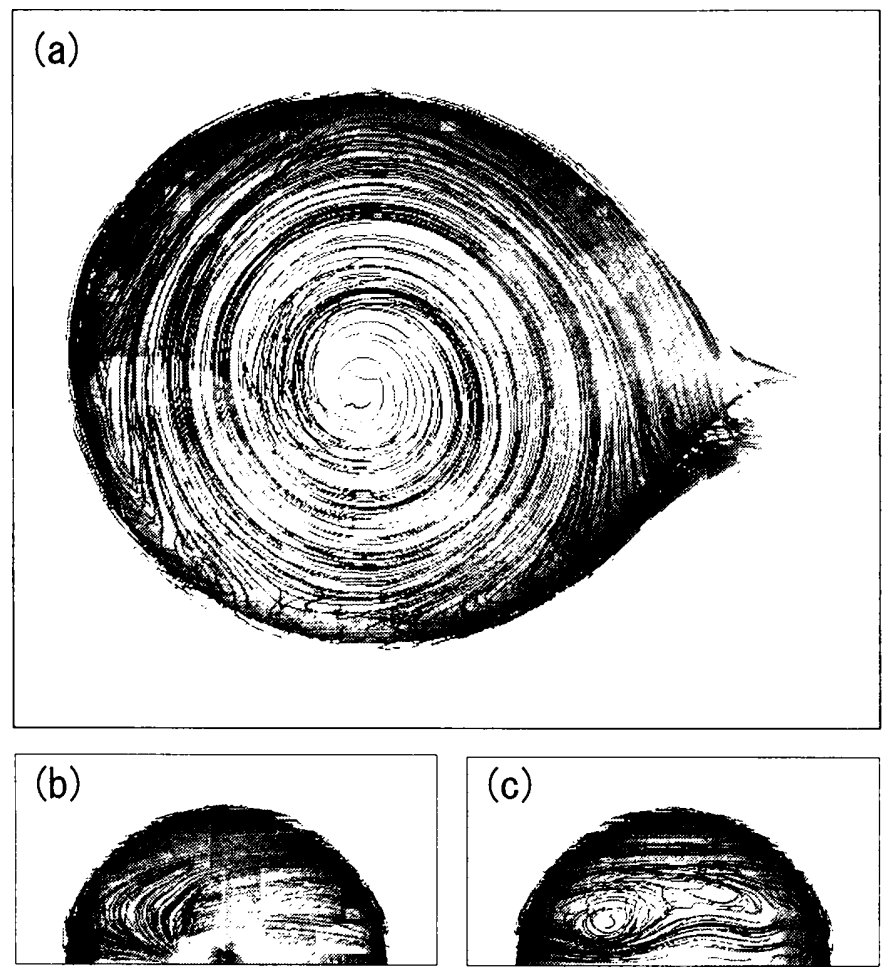

Fig. 1. The equi-density surface on the companion and the streamlines seen from (a) the north pole, (b) the L1point, and (c) the opposite side of the L1-point.

emission originates is still unknown, and 2) the companion's mass has still not been established. Here we try to attribute the He II $\lambda 4686$ lines to the irradiated spot on the surface of the companion and estimate the companion's mass via the Doppler mapping (Oka et al. 2003). The results show the mass of the companion to be $\sim 2 M_{\odot}$ assuming the mass of the white dwarf to be around $0.6 M_{\odot}$. The need for a massive companion is consistent with van den Heuvel (1992). Moreover, if our model is correct, the observed Doppler map (Deufel et al. 1999) reflects the surface flow on the companion.

\section{REFERENCES}

Deufel, B. et al. 1999, A\&A, 343, 455

Lubow, S.H. \& Shu, F.H. 1975, ApJ, 198, 383

Oka, K. et al. 2002, A\&A, 394, 115

Oka, K. et al. 2003, A\&A, submitted

van den Heuvel, E. P. J. et al. 1992, A\&A, 262, 97 